\title{
Searches for physics beyond the Standard Model in leptonic final states using the ATLAS detector
}

\author{
Paolo Mastrandrea*, on behalf of the ATLAS Collaboration \\ Università di Pisa \& INFN, Italy \\ E-mail: paolo.mastrandrea@cern.ch
}

\begin{abstract}
The ATLAS experiment at the Large Hadron Collider (LHC) pursues a wide and comprehensive program of searches for new physics Beyond the Standard Model (BSM). In this summary the results of two searches for new resonances in leptonic final states are presented. The data were recorded by the ATLAS experiment in proton-proton collisions at a centre-of-mass energy of $\sqrt{s}=13 \mathrm{TeV}$ during Run 2 of the LHC.

A search for a new heavy, charged $W^{\prime}$ boson decaying into an electron or muon and a neutrino was conducted using an integrated luminosity of $79.8 \mathrm{fb}^{-1}$. As no excess of events above the Standard Model prediction is observed, the results are used to set upper limits on the $W^{\prime}$ boson cross-section times branching ratio to an electron or muon and a neutrino as a function of the $W^{\prime}$ mass. Assuming a $W^{\prime}$ boson with the same couplings as the Standard Model $W$ boson, $W^{\prime}$ masses below $5.6 \mathrm{TeV}$ are excluded at the $95 \%$ confidence level.

A search for high-mass dielectron and dimuon resonances in the mass range of $250 \mathrm{GeV}$ to $6 \mathrm{TeV}$ was conducted using an integrated luminosity of $139 \mathrm{fb}^{-1}$. No significant deviation is observed, and upper limits are placed at the $95 \%$ confidence level on the fiducial cross-section times branching ratio for various resonance width hypotheses. For a set of benchmark models, the limits are converted into lower limits on the resonance mass and reach $4.5 \mathrm{TeV}$ for the $\mathrm{E}_{6}$-motivated $Z_{\psi}^{\prime}$ boson.
\end{abstract}

XXVII International Workshop on Deep-Inelastic Scattering and Related Subjects - DIS2019

8-12 April, 2019

Torino, Italy

${ }^{*}$ Speaker. 


\section{Introduction}

Extensions to the Standard Model (SM) may include heavy gauge bosons that could be discovered at the Large Hadron Collider (LHC) [2]. Conceptually, these particles are heavier versions of the Standard Model (SM) $W$ and $Z$ bosons and are generically referred to as $W^{\prime}$ and $Z^{\prime}$ bosons. Typical benchmark models include the Sequential Standard Model (SSM), which implements the same fermion couplings as the SM $W$ and $Z$ bosons, a $Z_{\chi}^{\prime}$ and a $Z_{\Psi}^{\prime}$ boson of an $E_{6}$-motivated Grand Unification model. The ATLAS experiment at the Large Hadron Collider (LHC) pursues a wide and comprehensive program of searches for new physics Beyond the Standard Model (BSM). In this report a search for a $W^{\prime}$ boson decaying in a charged lepton and a neutrino and a search for a $Z^{\prime}$ boson decaying in a pair of charged leptons are presented. In the following, the term lepton $(l)$ is used to refer to an electron or a muon.

\section{The ATLAS detector}

ATLAS [1] is a multipurpose detector with a forward-backward symmetric cylindrical geometry with respect to the LHC beam axis. The innermost layers consist of tracking detectors in the pseudorapidity range $|\eta|<2.5$. This inner detector (ID) is surrounded by a thin superconducting solenoid that provides a $2 \mathrm{~T}$ axial magnetic field. It is enclosed by the electromagnetic and hadronic calorimeters, which cover $|\eta|<4.9$. The outermost layers of ATLAS consist of an external muon spectrometer (MS) within $|\eta|<2.7$, incorporating three large toroidal magnetic assemblies with eight coils each. The field integral of the toroids ranges between 2.0 and $6.0 \mathrm{~T} \mathrm{~m}$ for most of the acceptance. The MS includes precision tracking chambers and fast detectors for triggering. A two-level trigger system reduces the recorded event rate to an average of $1 \mathrm{kHz}$.

\section{Lepton plus Missing Transverse Momentum channel}

A search for a $W^{\prime}$ boson was conducted in the $W^{\prime} \rightarrow l v$ channel using $79.8 \mathrm{fb}^{-1}$ of data collected by the ATLAS experiment for proton-proton ( $p p)$ collisions at the LHC at a centre-of-mass energy $\sqrt{s}=13 \mathrm{TeV}$ [3]. The analysis uses events with a high transverse momentum $\left(p_{\mathrm{T}}\right)$ lepton and significant missing transverse momentum $E_{\mathrm{T}}^{\text {miss }}$, which is used to infer the presence of the neutrino in the event as it escapes direct detection. The signal discriminant is the transverse mass, which is defined as $m_{\mathrm{T}}=\sqrt{2 p_{\mathrm{T}} E_{\mathrm{T}}^{\mathrm{miss}}\left(1-\cos \phi_{l v}\right)}$, where $\phi_{l v}$ is the azimuthal angle between the directions of the lepton $p_{\mathrm{T}}$ and the $E_{\mathrm{T}}^{\text {miss }}$ in the transverse plane. A high mass $W^{\prime}$ signal would appear as an excess of events above the SM background at high $m_{\mathrm{T}}$. The SM background mainly arises from processes with at least one prompt final-state lepton, with the largest source being the charged-current Drell-Yan (DY) $W$ boson production, where the $W$ boson decays into an electron or muon and a neutrino. The second largest source is top-quark pair $(t \bar{t})$ and single-top-quark production, denoted in the following as "top-quark background". Other non-negligible contributions are from the neutral-current DY $\left(Z / \gamma^{*}\right)$ process, diboson production, as well as from events in which one final-state jet or photon satisfies the lepton selection criteria. This last component of the background, referred to in the following as the multijet background, receives contributions from multijet, heavy-flavour quark and $\gamma+$ jet production. The multijet background is determined using 


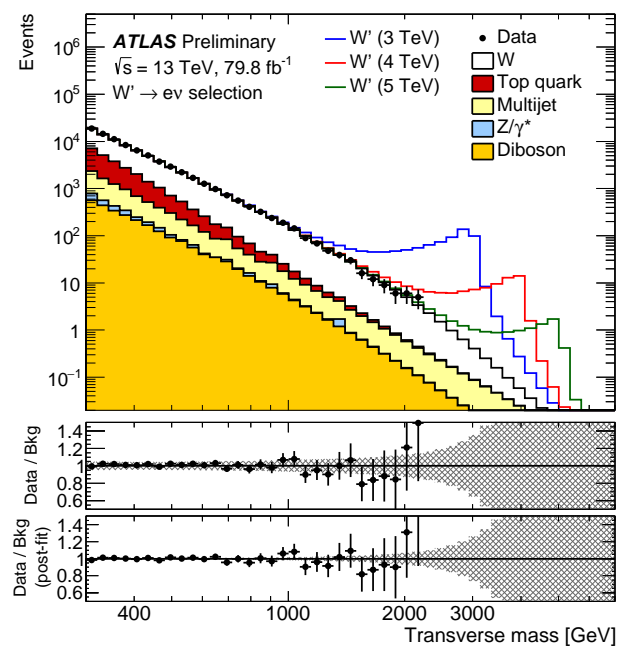

(a)

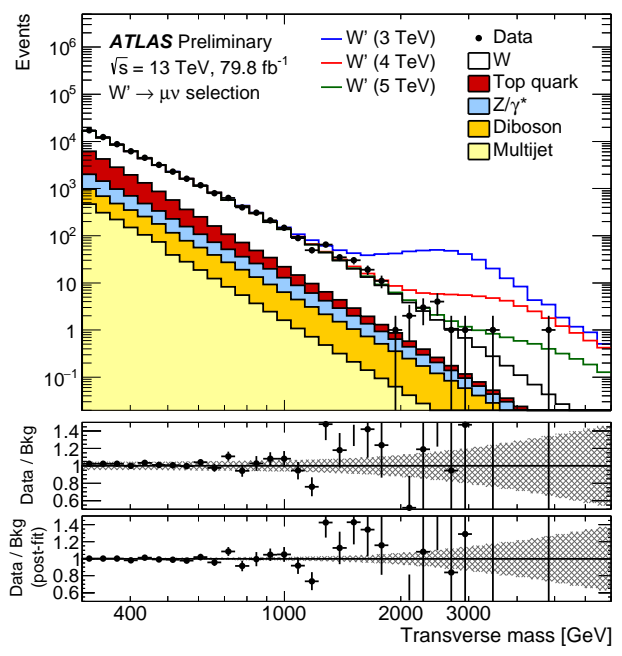

(b)

Figure 1: Transverse mass distributions for events satisfying all selection criteria in the (a) electron and (b) muon channels [3]. The distributions in data are compared to the stacked sum of all expected backgrounds. As examples, expected signal distributions for three different SSM $W^{\prime}$ boson masses are shown on top of the SM prediction. The middle panels show the ratios of the data to the expected background, with vertical bars representing both data and MC statistical uncertainties. The lower panels show the ratios of the data to the adjusted expected background that results from the statistical analysis. The bands in the ratio plots indicate the total systematic uncertainty.

a data-driven method, while the other backgrounds and the signal samples $\left(W^{\prime} \rightarrow e v\right.$ and $W^{\prime} \rightarrow \mu v$ ) are modelled by Monte Carlo (MC) simulations.

The compatibility between the data and the predicted background is evaluated with a profilelikelihood ratio test quantifying the probability that the background fluctuates to give a signal-like excess equal to or larger than what is observed. The likelihood functions in the ratio are products of Poisson probabilities over all bins in the transverse mass distribution (as shown in Figure 1) and log-normal constraints for the variations in signal and background yields associated with systematic uncertainties. In the denominator of the likelihood ratio, the likelihood function is maximised assuming the presence of a signal above the expected background, and in the numerator assuming the background-only hypothesis. To model the signal, $W_{S S M}^{\prime}$ templates are used for a series of $W_{S S M}^{\prime}$ masses in the search range $350 \mathrm{GeV} \leq m_{W^{\prime}} \leq 6000 \mathrm{GeV}$. Figure 1 displays a few examples of these templates. No significant excesses are observed in the data.

Based on the above findings, upper limits on the cross-section for producing a $W_{S S M}^{\prime}$ boson times its branching ratio to only one lepton generation $(\sigma \times B R)$ are computed at the 95\% CL as a function of the $W_{S S M}^{\prime}$ boson mass. The limits are calculated in a Bayesian analysis with a uniform positive prior probability distribution for $\sigma \times B R$. The observed upper limits are extracted by comparing data to the expected background and signal using $W_{S S M}^{\prime}$ templates for the same range of signal masses as for the profile-likelihood ratio test. The expected limits are derived 


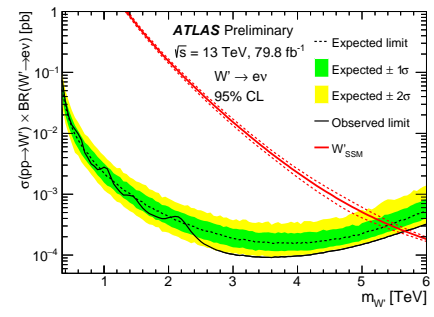

(a)

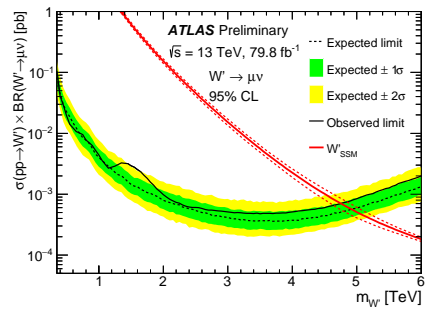

(b)

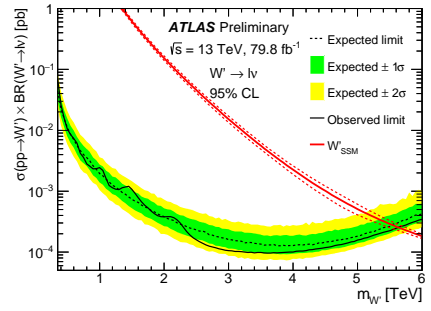

(c)

Figure 2: Observed (solid black line) and expected (dashed black line) upper limits on cross-section times branching ratio $(\sigma \times B R)$ as a function of the SSM $W^{\prime}$ boson mass in the (a) electron, (b) muon and (c) combined electron and muon channels [3]. The $1 \sigma$ (green) and $2 \sigma$ (yellow) expected limit bands are also shown. The predicted $\sigma \times B R$ for SSM $W^{\prime}$ production is shown as a solid red line. Uncertainties on the predicted $\sigma \times B R$ are shown as dashed red lines.

from pseudo-experiments obtained from the estimated background distributions. The median of the distribution of the limits from the pseudo-experiments is taken as the expected limit, and $1 \sigma$ and $2 \sigma$ bands are defined as the ranges containing respectively $68 \%$ and $95 \%$ of the limits obtained with the pseudo-experiments. The $95 \% \mathrm{CL}$ upper limits on $\sigma \times B R$ as a function of the $W_{S S M}^{\prime}$ mass are shown in Figure 2 separately for the electron and muon channels and for the combination of the two channels. The theoretical uncertainties and the uncertainty in the integrated luminosity are treated as correlated between the channels. Figure 2 also shows the predicted $\sigma \times B R$ for the $W_{S S M}^{\prime}$ boson as a function of its mass as well as the uncertainties from the PDF, $\alpha_{s}$ and the factorisation and renormalisation scales derived using the same prescription as used for the $W$ boson production. The observed (expected) lower mass limit for a $W_{S S M}^{\prime}$ boson, is 5.6 (5.5) $\mathrm{TeV}$ for the combination of the electron and muon channels.

\section{Dilepton channel}

A search for a new resonance decaying into two electrons or two muons was conducted using $139 \mathrm{fb}^{-1}$ of data collected by the ATLAS experiment for proton-proton ( $p p$ ) collisions at the LHC at a centre-of-mass energy $\sqrt{s}=13 \mathrm{TeV}$ [4]. A resonant signal is searched for by fitting the data dilepton mass distribution. The fit function consists of a smooth functional form for the background, and a generic signal shape. The generic signal shapes are constructed from nonrelativistic Breit-Wigner functions of various widths convolved with the detector resolution. If the width of a signal resonance is negligible (zero-width) compared to the detector resolution, then the measured dilepton invariant mass distribution is obtained from the mass resolution only. To allow for a generic resonance search, a fiducial region at particle level is defined following the selection criteria applied to the reconstructed lepton candidates and the additional criteria for the dilepton mass $m_{l l}^{\text {true }}>m_{X}-2 \Gamma_{X}$, where $m_{X}$ and $\Gamma_{X}$ represent the pole mass and width of a hypothetical resonance $X$, respectively. This selection is added in order to reduce the model dependence from off-shell effects. 


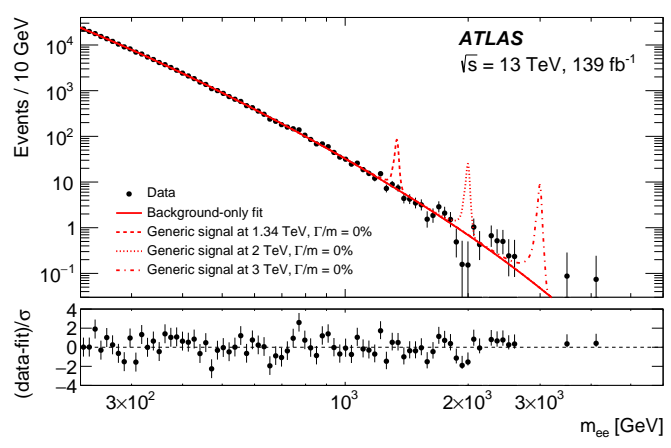

(a)

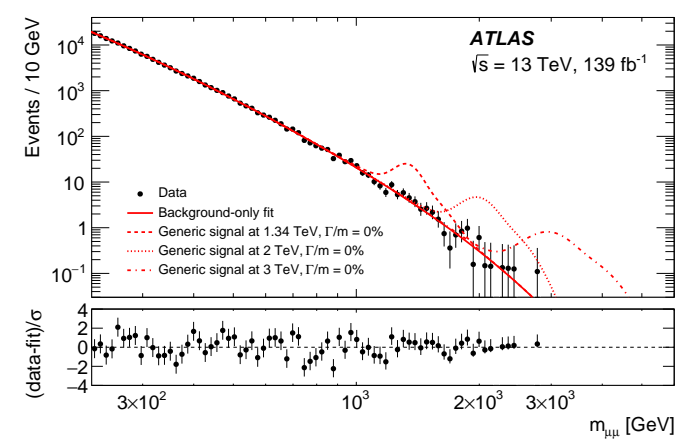

(b)

Figure 3: Distribution of the (a) dielectron and (b) dimuon invariant mass for selected events. Generic zero-width signal shapes, scaled to 20 times the value of the corresponding expected upper limit at 95\% CL on the fiducial cross-section times branching ratio, with pole masses of $m_{X}=1.34$, 2 and $3 \mathrm{TeV}$ (for illustration purposes) as well as background-only fits are superimposed [4]. The error bars indicate statistical uncertainties only. The differences between the data and the fit results in units of standard deviations of the statistical uncertainty are shown in the bottom panels.

The smooth functional form for the background is based on fit performance studies on a MC background template. The amount of signal due to the residual disagreement between the functional form and the background distribution ("spurious signal") is also estimated through these studies. In order to minimise the statistical uncertainties in this procedure, the background template for DY is produced from large-statistics samples simulated only at generator level and smeared by the experimental dilepton mass resolution, with mass-dependent acceptance and efficiency corrections applied. A similar procedure is applied to the generator-level dilepton mass distribution in the $t \bar{t}$ sample exploiting the larger number of events from the generator-level mass distribution. The distributions from the diboson and single-top simulated samples and, in the electron channel, a template for multi-jet and $W+$ jet processes are also considered. Uncertainties related to the background modelling are propagated into the determination of the spurious signal.

The numbers of signal and background events, as a function of the signal mass and width hypothesis, are estimated from simultaneous maximum-likelihood fits of the signal-plus-background models to the data $m_{l l}$ distribution. Systematic uncertainties are included in the fits via nuisance parameters constrained by penalty terms which are either Gaussian (e.g. energy and momentum scale uncertainties) or log-normal (efficiency and resolution uncertainties). Potential mismodelling of the background estimate is accounted for through an additional nuisance parameter allowing nonzero signal normalisation under the null hypothesis constrained by the measured spurious signal. Dielectron and dimuon channels are considered both as independent channels and in a combined approach, under a lepton-flavour universality assumption.

The probability that the data are compatible with the background-only hypothesis is shown in Figure $4 \mathrm{a}$ as a function of pole mass for zero-width signals. No significant excess is observed.

Figure $4 \mathrm{~b}$ shows the upper limits on the fiducial cross-section times branching ratio to two 


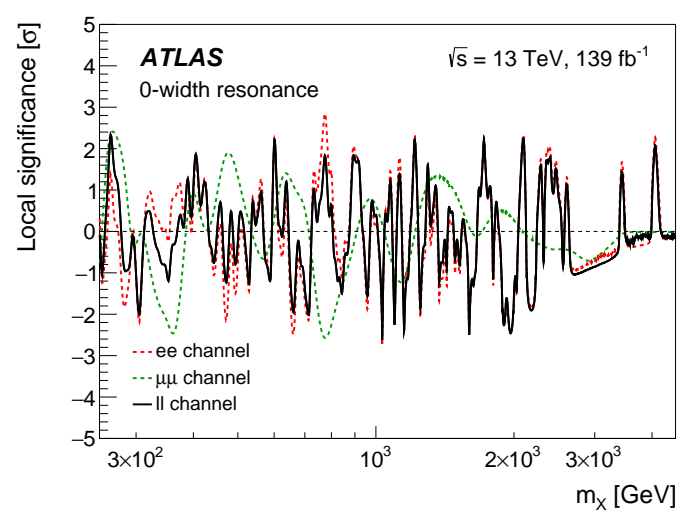

(a)

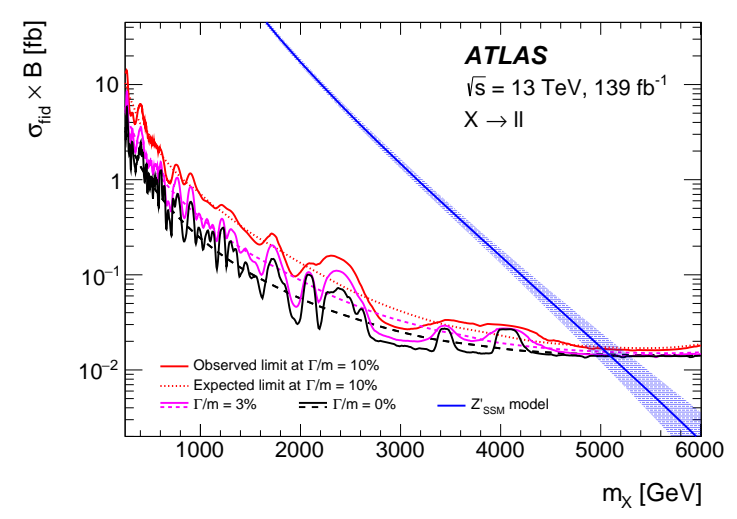

(b)

Figure 4: (a) Probability that the observed spectrum is compatible with the background-only hypothesis for the dielectron, dimuon and combined dilepton channels [4]. The local $p_{0}$ is quantified in standard deviations $\sigma$ as a function of pole mass $m_{X}$. (b) Upper limits at 95\% CL on the fiducial cross-section times branching ratio as a function of pole mass for the zero-width, 3\%, 10\% relative width signals for the combined dilepton channel [4]. Observed limits are shown as a solid line and expected limits as a dotted/dashed line. The signal theoretical uncertainties are shown as a band on the $Z_{S S M}^{\prime}$ theory line.

electrons and two muons for generic resonances of various relative widths as a function of their mass. The observed limit on the fiducial cross-section times branching ratio ranges from 3.6 (13.1) $\mathrm{fb}$ at $250 \mathrm{GeV}$ to about $0.014(0.018) \mathrm{fb}$ at $6 \mathrm{TeV}$ for the zero (10\%) relative width signal in the combined dilepton channel. The generic cross-section limits at $\Gamma / \mathrm{m}=0.5 \%, 1.2 \%$ and $3.0 \%$ are compared with the model predictions of $Z_{\Psi}^{\prime}, Z_{\chi}^{\prime}$ and $Z_{S S M}^{\prime}$, respectively, to obtain mass limits. The cross-section values for the model predictions are obtained in the fiducial volume, for compatibility with the definition of the generic signal model. Mass limits are calculated as the intersection between the expected and observed limits with the model prediction. In particular, the results imply a lower limit of $4.5(5.1) \mathrm{TeV}$ on $m_{Z^{\prime}}$ for the $Z_{\Psi}^{\prime}\left(Z_{S S M}^{\prime}\right.$ ) boson at $95 \%$ confidence level.

\section{References}

[1] ATLAS Collaboration, JINST 3 (2008) S08003.

[2] L. Evans and P. Bryant, JINST 3 (2008) S08001.

[3] ATLAS Collaboration, ATLAS-CONF-2011-068, http://cdsweb.cern.ch/record/1349309.

[4] ATLAS Collaboration, arXiv:1903.06248. 